\title{
A Century of Shock and Wave Dynamics
}

\author{
Sir James Lighthill \\ Department of Mathematics, University College London, Gower St, London WC1E 6BT, UK
}

\begin{abstract}
The recent three-volume history 'Twentieth Century Physics' ${ }^{1}$ includes my 118 page 'Chapter 10. Fluid Dynamics' about a field where the 20th century's first decade saw big breakthroughs in the analysis of nonlinear problems for which the physicist's standard perturbation methods break down - and which would later be named singular perturbation problems. Besides the 1904 elucidation of boundary-layer structure by Prandtl, these included the 1910 elucidation of shock-wave structure in complementary investigations by Rayleigh and by Taylor. Subsequent advances in shock wave dynamics brought crucial new discoveries on the structure and propagation of weak shocks, and also on shock-wave/boundary-layer interaction, both with major aeronautical implications; along with still harder investigations into the structure and propagation of strong shock waves such as appear in explosions and implosions and also around spacecraft re-entering the earth's atmosphere. It may, perhaps, be worth noting that questions which remain relatively simple for weak waves, like the nature of reflexion and diffraction by a solid body, raise formidable and intriguing difficulties for strong shock waves.

In this introductory paper looking back over a century of shock wave dynamics, I highlight (i) key analytical approaches for both weak and strong shock waves, (ii) beautiful and effective optical methods for use in wind tunnels and shock tubes and (iii) powerful techniques for accurate shock capturing in computational fluid dynamics.
\end{abstract}

\section{INTRODUCTION}

A century ago, students of Acoustics and Vibration depended above all on Rayleigh's superb treatise 'The Theory of Sound' which had just appeared in an expanded second edition ${ }^{2}$. Yet in section 253 of this great work Rayleigh brilliantly showed how consideration of just the simplest problem in the nonlinear theory of sound posed an enigma which it was quite impossible to resolve with the knowledge then available. Existing suggestions for a resolution, incorporating those discontinuous waves or shock waves which Mach ${ }^{3}$ had photographed around bullets in flight, appeared on Rayleigh's analysis to contradict fundamental physical principles; in short, the 'century of shock wave dynamics' had not yet begun.

In surveying the dynamics (rather than the whole physics) of shock waves, space may be saved by concentrating on waves propagated through a perfect gas with constant specific heats in a ratio $\gamma$; for which scientists since Laplace ${ }^{4}$ had appreciated how, in any sound wave, those changes of pressure $p$ and density $\rho$ whose ratio is the square of the sound speed $c$ must satisfy the adiabatic relationship

$$
\frac{p}{p_{0}}=\left(\frac{\rho}{\rho_{0}}\right)^{\gamma} \text { giving } c^{2}=\frac{d p}{d \rho}=c_{0}^{2}\left(\frac{\rho}{\rho_{0}}\right)^{\gamma-1} \text { with } c_{0}^{2}=\frac{\gamma p_{0}}{\rho_{0}} .
$$

(Here, subscript zero denotes undisturbed values.) On linear theory sound is propagated at speed $c_{0}$, whereas on nonlinear theory higher pressures travel at an increased sound speed $c$, amounting on a first approximation to

$$
c=c_{0}+\frac{\gamma-1}{2}\left(\frac{p-p_{0}}{\rho_{0} c_{0}}\right)=c_{0}+\frac{\gamma-1}{2} u,
$$

where $u$, the gas velocity in the direction of propagation, assumes on a linear approximation the familiar bracketed form. But this propagation speed Eq. (2), relative to a fluid which itself moves at velocity $u$, implies an absolute velocity

$$
c+u=c_{0}+\frac{\gamma+1}{2} u
$$

of wave travel. (For air, with $\gamma=1.4$, the excess wave speed is $1.2 u$, out of which just one-sixth arises from the increase Eq. (2) in $c$ while five-sixths is due to convection of sound at the air velocity $u$.)

Although expression (3) for the wave speed is introduced above by crude approximate arguments, Riemann's subtle mathematical analysis of 1859 had already proved it to be absolutely accurate ${ }^{5}$ for plane sound waves of any amplitude propagated one-dimensionally into undisturbed fluid under adiabatic conditions. Briefly, the relationship $c=c_{0}+\frac{1}{2}(\gamma-1) u$ is exact; the expressions for pressure and density may be derived from $c$ by Eqs. (1); and, most important of all, each value of $u$ is propagated at precisely the speed Eq. (3).

These conclusions were well known to Rayleigh, who recognised also their sensational implications. Figure 1 shows these in the case of a single pulse of positive excess pressure, represented as an initial graph (solid line) of fluid velocity $u$ against distance. On a linear theory of one-dimensional sound waves, each value of $u$ would be propagated at speed $c_{0}$, so that the shape of the pulse would remain unchanged when plotted (as here) against $x-c_{0} t$. On the exact nonlinear theory, however, each value of $u$ is propagated at speed $c_{0}+\frac{1}{2}(\gamma+1) u$; accordingly, after time $t$, that value when plotted against $x-c_{0} t$ as in Fig. 1 has been shifted a distance $\frac{1}{2}(\gamma+1) u t$ to the right. Small values of $u$ have hardly moved at all, while large values have moved much more - allowing them, remarkably, to 'catch up' with smaller values.

These distorted pulse shapes are shown in Fig. 1 (broken lines) for a sequence of values of $t$ until a time has been reached when the pulse shape has a vertical tangent. Pulse 